\title{
Quality of life and stability of tooth color change at three months after dental bleaching
}

\author{
Cristian Bersezio ${ }^{1,2}$. Javier Martín ${ }^{1} \cdot$ Carla Mayer $^{1} \cdot$ Oriana Rivera $^{1} \cdot$ Juan Estay ${ }^{1} \cdot$ Rolando Vernal $^{3,6}$. \\ Ziyad S. Haidar ${ }^{4,5} \cdot$ Pablo Angel $^{1} \cdot$ Osmir B. Oliveira Jr. ${ }^{2} \cdot$ Eduardo Fernández $^{1,6}{ }^{1}$
}

Accepted: 16 August 2018 / Published online: 21 August 2018

(c) Springer Nature Switzerland AG 2018

\begin{abstract}
Purpose Intracoronary bleaching is a minimally invasive, alternative treatment that addresses aesthetic concerns related to non-vital teeth discoloration. However, to the best of our knowledge, no studies have assessed the psychosocial impacts of such procedures on patients' aesthetic perceptions. The aim of this study was to evaluate aesthetic perceptions and the psychosocial impact of patients up to 3 months after their teeth had been bleached with hydrogen peroxide (35\%) and carbamide peroxide (37\%) using the walking bleach technique.

Methods The patients were randomly divided into two groups according to the bleaching agent used: G1 =hydrogen peroxide $35 \%(n=25)$ and $\mathrm{G} 2=$ carbamide peroxide 37\% $(n=25)$. Non-vital bleaching was performed in four sessions. Color was objectively $(\Delta \mathrm{E})$ and subjectively $(\Delta \mathrm{SGU})$ evaluated. Aesthetic perception and psychosocial factors were evaluated before, 1 week and 1 month after the bleaching using the Oral Health Impact Profile (OHIP) and Psychosocial Impact of Dental Aesthetics Questionnaire (PIDAQ) questionnaires.

Results The color change $(\Delta \mathrm{E})$ values at 1 month were $\mathrm{G} 1=16.80 \pm 6.07$ and $\mathrm{G} 2=14.09 \pm 4.83$. These values remained stable until the third month after treatment $(p>0.05)$. There was a decrease in the values of OHIP-aesthetics and PIDAQ after treatment versus baseline $(p<0.05)$. This status was maintained through the third month after treatment.

Conclusions Both agents were highly effective and had a positive impact on the aesthetic perception and psychosocial impact of patients, values that also remained stable over time. Non-vital bleaching yields positive and stable impacts on aesthetic perception and psychosocial factors. ClinicalTrials.gov identifier NCT02718183.
\end{abstract}

Keywords Dark tooth bleaching $\cdot$ Psychosocial impact $\cdot$ Aesthetic auto-perception $\cdot$ Randomized clinical trial

Electronic supplementary material The online version of this article (https://doi.org/10.1007/s11136-018-1972-7) contains supplementary material, which is available to authorized users.

Eduardo Fernández

edofdez@yahoo.com

1 Department of Restorative Dentistry, Faculty of Dentistry, University of Chile, Independencia, Santiago, Chile

2 Department of Restorative Dentistry, School of Dentistry, University Estadual Paulista-UNESP, Araraquara CEP 14801-903, Brazil

3 Departament of Conservative Dentistry, Faculty of Dentistry, University of Chile, Independencia, Santiago, Chile

\section{Background}

One of the most critical aspects of an aesthetically pleasing smile is tooth color. When a single tooth presents a color change, the adverse effect may be more pronounced than when a generalized (full dentition) color change persists because it is evident that the color of one tooth differs from

4 BioMAT'X, Centro de Investigación Biomédica, Facultad de Medicina, Universidad de los Andes, Av. Mons. Álvaro del Portillo, 12.455 Las Condes, Santiago, Chile

5 Faculty of Dentistry, Universidad Los Andes, Av. Mons. Álvaro del Portillo, 12.455 Las Condes, Santiago, Chile

6 Instituto de Ciencias Biomédicas, Universidad Autónoma de Chile, Av. Pedro de Valdivia 425, Providencia, Santiago, Chile 
that of the rest of the teeth [1]. Intracoronary (inside-thetooth) bleaching is a widely used and minimally invasive alternative treatment/technique to solve aesthetic non-vital (endodontically treated tooth $=$ replacing the live tissue inside the tooth with a filling) tooth discoloration (i.e., "dark teeth") [2]. Intracoronary bleaching has been reported to have a high rate of patient satisfaction [3]. To the best of our knowledge, no studies have assessed the real impact of such procedures on the aesthetic perception of patients. However, recent studies using OHIP and OIDP have reported positive effect(s) on QoL (effect size $=0.38$ [4], 0.48 [5] and 0.27 [6]), on patients undergoing complete denture bleaching techniques applied to vital teeth [4-6].

The most common dental bleaching agents are hydrogen peroxide and carbamide peroxide. These agents are used in high concentrations for non-vital teeth and act as oxidizing organic pigments via the decomposition products of the chemical agent $[2,7]$.

The primary objective of this study was to evaluate the psychosocial impact and aesthetic self-perception of patients undergoing non-vital tooth bleaching. This study also compared the stability of the color change obtained 3 months after the bleaching treatment, which involved either hydrogen peroxide $(35 \%)$ or carbamide peroxide $(37 \%)$ gels applied using the walking bleach technique. Our hypotheses were as follows: (1) There would be a positive impact on quality of life 3 months after treatment in patients who received non-vital bleaching using the walking bleach technique either with $35 \%$ hydrogen peroxide or $37 \%$ carbamide peroxide; and (2) There would be stability in the color (tone) change of non-vital bleached teeth using either 35\% hydrogen peroxide or $37 \%$ carbamide peroxide 3 months after the treatment.

\section{Methods}

This randomized clinical study and its protocols were approved by the Ethics Committee of the Faculty of Dentistry, University of Chile (2016/04) and was conducted according to the Consolidated Standards of Reporting Trials Statement [8] and the Declaration of Helsinki [9]. The http://www.ClinicalTrials.gov identifier is NCT02718183.

A randomized, double-blind study (patients and evaluator) was designed, and simple method of randomization was performed using Excel 2013 software (Microsoft, Seattle, Washington, USA). Patients were recruited via flyers distributed in a dental school and via social media outlets such as Facebook and Twitter.

\section{Sample size}

The sample size was determined using GPower 3.1 [10] software with a 5\% level of significance, $90 \%$ statistical power and a drop-out rate of $25 \%$ based on a previous study [11]. This study corresponds to a therapeutic equivalence type where a color variation of $\Delta \mathrm{E}$ (Euclidean distance or color difference between baseline and follow-up) tones in the equivalence range of $7-10$, based on original color, was considered to be significant. We targeted a sample size of 20. However, to compensate for the drop-out rate reported in previous studies, we included 25 patients per group.

Forty-seven patients were selected initially with at least one non-vital tooth discoloration of A2 or more according to the Vita Classical dental color shade guide (Vita Zahnfabrik, Bad Säckingen, Germany) scale. All of the subjects accepted and signed an informed consent form prior to the start of the clinical trial.

\section{Selection criteria}

\section{Inclusion criteria}

Patients over an age of 18 years who had one or more nonvital, discolored incisor with an A2 or higher value according to the Vita Classical scale. Restoration(s), if present, did not include the vestibular surface. When present, endodontic treatment had to be in good condition (adequately filled in amplitude and length and asymptomatic), the patients had to have not undergone bleaching previously.

\section{Exclusion criteria}

Patients who were pregnant or lactating, patients with cancer, patients with enamel defects (enamel hypoplasia or fluorosis) or dental staining by tetracycline or metallic pigments or amalgam derivatives. We also excluded patients in orthodontic treatment with fixed appliances, patients with periodontal disease, teeth with carious lesions or periapical pathology and/or dental resorption (external or internal).

Patients identified with any pathology were referred to the appropriate clinical treatment within the dental school.

Patients who met the study criteria and agreed to participate were randomly selected and divided into two parallel study groups according to the bleaching used:

$\mathrm{G} 1=35 \%$ hydrogen peroxide (Opalescence EndoUltradent, South Jordan, Utah, USA).

$\mathrm{G} 2=37 \%$ carbamide peroxide (Whiteness Superendo,

FGM, Joinville, Santa Catarina, Brazil). 


\section{Bleaching protocol}

An ambulatory technique (walking bleaching in which the bleaching gel remains in the teeth for a period of time) was used. The bleaching agent was applied in the pulp chamber (into the teeth) and the cavity was then sealed. The agent was changed/replaced every week for up to 4 weeks of treatment.

\section{Preparation session}

The root canal was prepared under absolute isolation (Rubber Dam Ash, Dentsply, Brazil, SP). The endodontic filling was removed $3 \mathrm{~mm}$ below the cement-enamel junction and then sealed mechanically with a resin-reinforced glass ionomer (Riva light cure, SDI, Bayswater, Victoria, Australia) with a thickness of $2 \mathrm{~mm}$. The cement was then light-cured for $60 \mathrm{~s}$ at a distance of $1 \mathrm{~cm}$ using a Raddi Cal lamp (SDI, Bayswater, Australia). Radiographic control was used to confirm proper sealing of the root canal.

\section{Four bleaching sessions}

Application of the bleaching agent was performed according to the manufacturer's instructions. The gel was left in the pulp chamber (inside the tooth) with moisture. The cavity was sealed between sessions with temporary cement (Fermin, Detax, Baden-Württemberg, Germany), and the gel was replaced every 7 days. At the end of the fourth week of bleaching, the access cavity was washed with water and temporarily sealed for 7 days. Then, the cavity was restored definitively using the resin-based composite Z350 XT (3M ESPE, St. Paul, Minnesota, USA).

Color measurements were made before the commencement of treatment (baseline), immediately after each of the four bleaching sessions, 1 week after treatment (pre- and post-restoration), and 1 and 3 months post-bleaching.

\section{Color evaluation}

\section{Objective assessment}

Two evaluators measured tooth color on the middle-third of the labial surface using a spectrophotometer [Vita Easyshade Compact (VITA Zahnfabrik, Bad Säckingen, Germany)]. To standardize this evaluation, a silicone matrix was made (Zetaplus, Zhermack, Rovigo, Italy) with a 6-mm-diameter window on the buccal surface that enabled the tip of the spectrophotometer to be positioned. Color change was determined using the CIELab system with the parameters $L^{*}, a^{*}$, and $b^{*}$. The color difference $(\Delta E)$ with respect to baseline was calculated for every control session. $\Delta \mathrm{E}$ was calculated using the following formula: $\Delta \mathrm{E}=[(\Delta \mathrm{L} *) 2+(\Delta \mathrm{a} *) 2+(\Delta \mathrm{b} *) 2] 1 / 2$.

\section{Oral health impact profile questionnaire (OHIP-aesthetics)}

To evaluate the aesthetic perception of patients, the OHIPaesthetics questionnaire was translated and validated in Chilean Spanish [12]. The questionnaire was applied at the beginning (baseline) and after 1 week, 1 month, and 3 months. The questionnaire consisted of 14 questions whose answers were scored according to a Likert scale. The score on each question ranged from 0 to 4 (never $=0$, almost never $=1$, occasionally $=2$, fairly often $=3$, very often $=4$ ).

\section{The psychosocial impact of dental aesthetics questionnaire}

The PIDAQ questionnaire [13, 14] contains 23 items divided into four subscales (one positive and three negative). The subscales correspond to the following dimensions: (1) selfconfidence based on dental appearance, (2) social impact, (3) psychosocial impact, and (4) aesthetic concern. Selfconfidence based on dental appearance consists of six items. The second dimension, social impact, contains eight items on the social aspects of the quality-of-life questionnaire. The third dimension, psychosocial impact, contains six items primarily related to the psychosocial impact of dental aesthetics. The fourth dimension is aesthetics and contains three items. The questionnaire is patient-filled and administered at the beginning of the session. It uses a five-point Likert scale that ranges from 0 (no impact of dental aesthetics in quality-of-life) to 4 (maximum impact of dental aesthetics in quality-of-life) for each element. The response options are as follows: not at all $=0$, a little $=1$, somewhat $=2$, strongly $=3$, and very strongly $=4$. The questionnaire was also validated in Spanish and had a reliability coefficient of 0.90 as measured by Cronbach's alpha [14]. Likewise, the questionnaire was applied at the beginning (baseline) and 1 week, 1 month, and 3 months after bleaching.

\section{Statistical analysis}

After verifying the normality of the data and the homogeneity of the variance-covariance matrix, we evaluated the treatment efficacy by comparing the color variation (E), which we analyzed using the Wilcoxon test for within-group comparisons and the Mann-Whitney test for between-group comparisons. We used the MANCOVA test to measure the differences according to the color components $\mathrm{L}$, $\mathrm{a}$ and $\mathrm{b}$ at 3 months using the baseline color as a covariant. The statistical analyses were performed using SPSS 23.0 (SPSS Inc., Chicago, IL, USA) with $\alpha=.05$. To compare the 
OHIP-aesthetics and PIDAQ questionnaire scores, we used the Wilcoxon test.

\section{Results}

Forty-seven patients with 50 non-vital discolored teeth were recruited. After 3 months, 42 patients completed the treatment; these individuals constituted our cohort. A final sample of 44 non-vital teeth was obtained (Fig. 1). The characteristics of the final sample are showed in Table 1. All of the statistical analyses were performed with data imputation for missing outcomes [intention-to-treat (ITT)] and without data imputation (per-protocol). In all of the analyses, the same overall conclusions were obtained (data not shown), in this study it was used the ITT form.

\section{Objective assessment of color}

Statistically significant differences were identified using the Mann-Whitney test for the two groups at all times in the $\Delta \mathrm{L}$ parameter $(p \leq 0.028)$ except for the control at the third month post-bleaching $(\rho=0.072)$. Table 2 lists the $\Delta \mathrm{E}$ values. The efficacy was similar between the groups $(\rho \geq 0.065)$. Both groups exhibited high effectiveness (i.e., at least 14 units of $\Delta \mathrm{E}$ on average the first month after bleaching). Color remained stable at the third month (Wilcoxon test; $>0.05$ between the first and the third month post-bleaching). Figure 2 illustrates the color

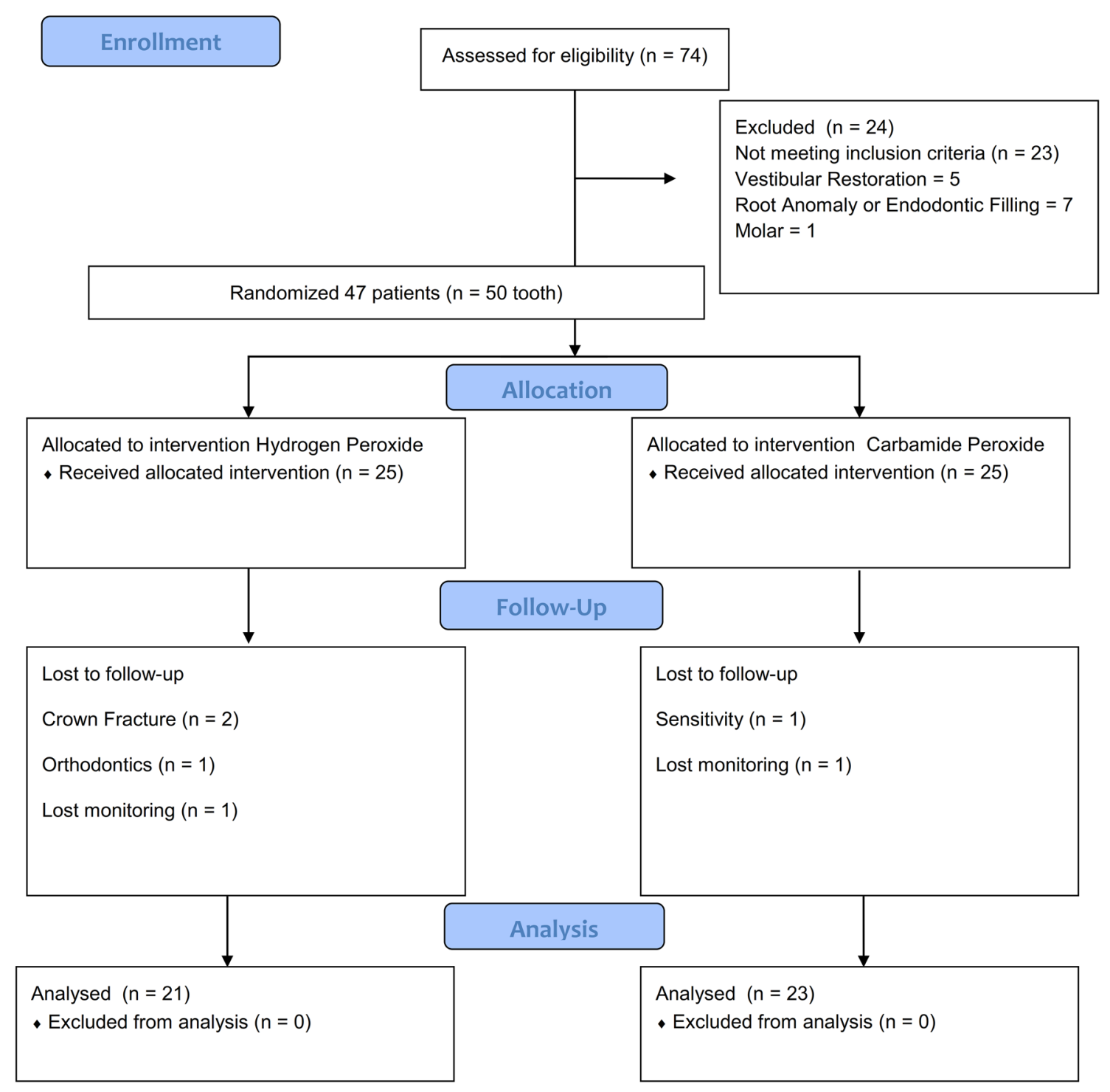

Fig. 1 Flow diagram of the clinical trial, including detailed information on the excluded participants 
Table 1 Participant baseline characteristics

\begin{tabular}{llll}
\hline Baseline features & Groups & Chi square test \\
\cline { 2 - 3 } & $\begin{array}{l}\text { Hydrogen peroxide } \\
(n=21)^{* *}\end{array}$ & $\begin{array}{l}\text { Carbamide peroxide } \\
(n=23)^{* *}\end{array}$ & \\
\hline Age (years; mean \pm SD) & $30.24 \pm 12.26$ & $30.83 \pm 11.25$ & 0.922 \\
Minimum age (years) & 19 & 20 & \\
Maximum age (years) & 65 & 65 & 0.823 \\
Male $(\%)$ & 42.9 & 39.1 & 0.09 \\
Trauma $(\%)$ & 52.38 & 39.13 & 0.871 \\
$\mathrm{~L}^{*}($ mean $\pm \mathrm{SD})$ & $73.29 \pm 8.65$ & $75.91 \pm 6.81$ & 0.921 \\
$\mathrm{a}^{*}($ mean $\pm \mathrm{SD})$ & $4.84 \pm 3.12$ & $4.85 \pm 3.39$ & 0.432 \\
$\mathrm{~b}^{*}($ mean $\pm \mathrm{SD})$ & $29.44 \pm 3.61$ & $31.79 \pm 6.60$ & \\
\hline
\end{tabular}

$S D$ standard deviation, * $(p>0.05)$, **number of patients assessed at 3-month follow-up. $L^{*}, a^{*}$ and $b^{*} . L^{*}$ stands for the lightness, and $a^{*}$ and $b^{*}$ for the green-red and blue-yellow axis of the CIElab color space, respectively
Table 2 Color change expressed in $\Delta \mathrm{E}$ units (mean and standard deviation) at all time points

\begin{tabular}{llrl}
\hline Assessment times & Color change by $\Delta \mathrm{E}$ & \multirow{2}{*}{ Mann-Whitney } \\
\cline { 2 - 3 } & G1=Hydrogen peroxide & $\begin{array}{c}\text { G2=Carba- } \\
\text { mide peroxide }\end{array}$ & \\
\hline Baseline versus 1-week bleaching & $10.08 \pm 4.83$ & $7.24 \pm 4.79$ & 0.065 \\
Baseline versus 1 month after bleaching & $16.04 \pm 4.88$ & $14.09 \pm 4.83$ & 0.307 \\
Baseline versus 3 month after bleaching & $15.25 \pm 5.28$ & $13.71 \pm 4.58$ & 0.503 \\
\hline
\end{tabular}

Statistically significant difference intragroup (Wilcoxon test, $p<0.05$ ) versus previous time point

\section{OHIP-aesthetics}

\section{Changes of color of non-vital tooth (1.1) submitted to walking bleaching technique}

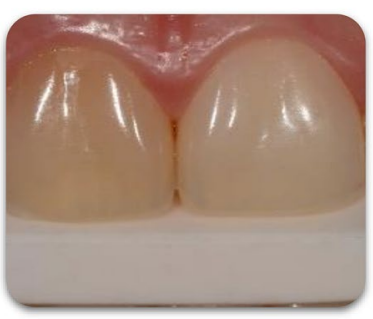

Before bleaching

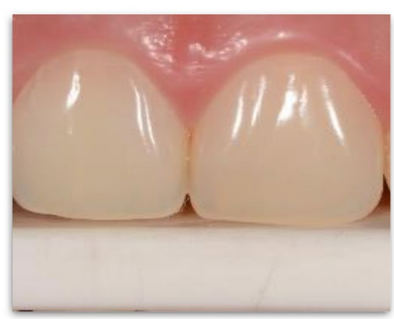

3 months post-bleaching
Fig. 2 Case that represents an ideal case of matching the color with a bleaching non-vital technique

change obtained with the walking bleaching technique and equalization with the homologous and neighboring teeth.

There were no differences between the components of the color $\mathrm{L}(p=0.72)$, a $(p=0.229)$ or $\mathrm{b}(p=0.251)$ axis between the groups according to the MANCOVA test at the third month of follow-up when was used the baseline color as a covariate.
The results of an OHIP-aesthetics questionnaire are listed in Table 3. No statistical differences were noted between the two groups $(\rho \geq 0.564)$. The functional limitation, physical pain, and psychological discomfort dimensions were significantly different $(\rho \leq 0.045)$ from the baseline values. The sum of values was also significantly different compared with the baseline $(p \leq 0.032)$.

\section{PIDAQ}

The PIDAQ questionnaire values, listed in Table 4, were significantly different between the baseline measurements and the 1-week, 1-month and 3-month post-treatment controls ( $\rho \leq 0.03$; Wilcoxon test). The one exception was the dimension of dental self-confidence in the hydrogen peroxide group (G1) at the third month $(\rho=0.54)$. When we compared the 1-week and 1-month post-treatment controls, the only statistically significant difference was in the aesthetic concern $(\rho \leq 0.42)$. This situation was evident for both groups.

Within the first and the third month post-treatment, there was a significant difference in the dimension of psychosocial impact for the hydrogen peroxide group (G1) $(p=0.43)$ as 


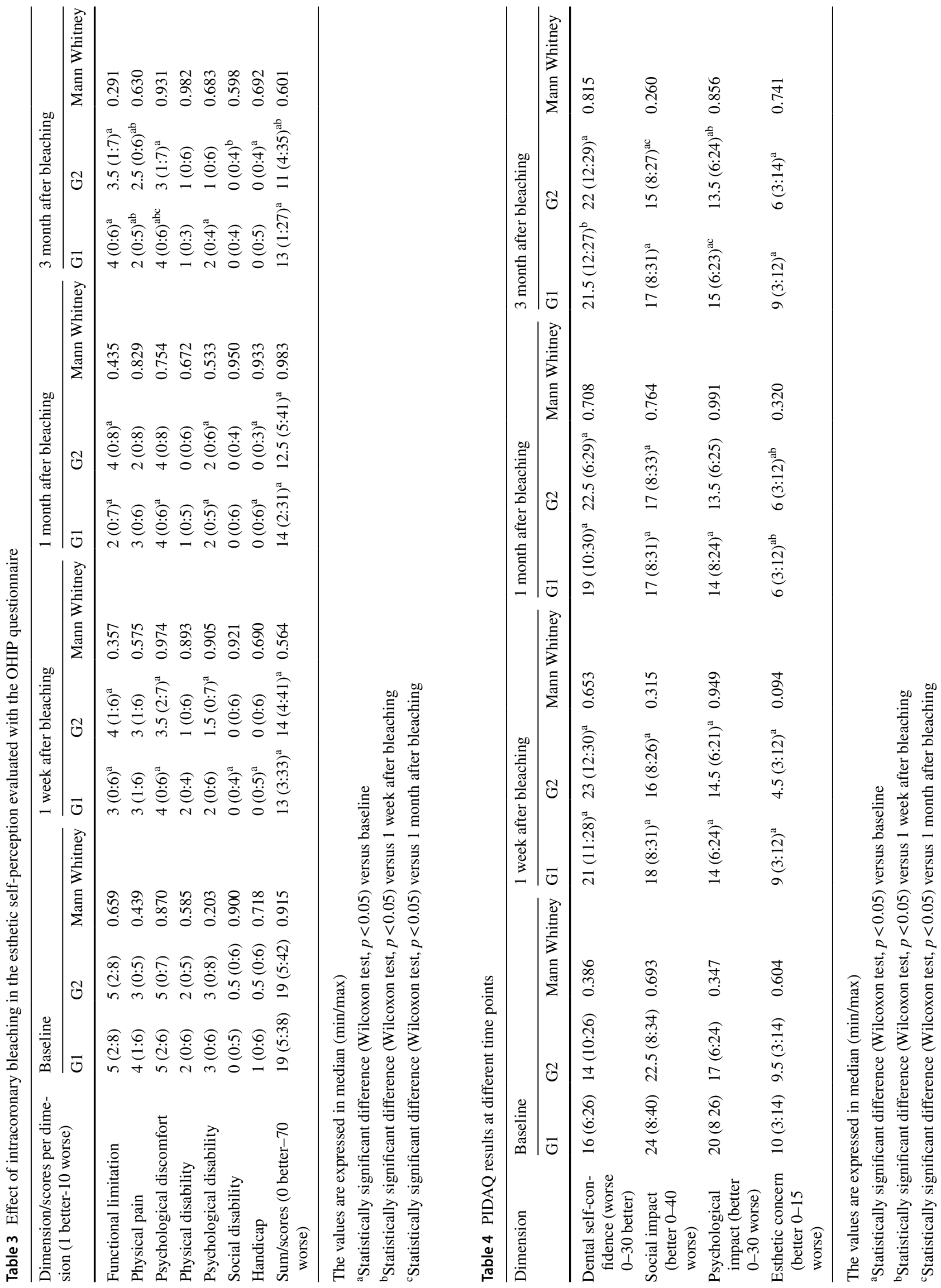


well as in social impact for the carbamide peroxide group $(\mathrm{G} 2)(\rho=0.047)$.

\section{Discussion}

Tooth bleaching alters the color of dental tissues via chemical catalysis (an oxidation-reduction reaction with the darkened substrate). In other words, the whitening effect is a result of the release of reactive oxygen from the degrading bleaching chemical agent upon introduction into the pulp chamber. This randomized, double-blind clinical study provides insights into aesthetic perception and psychosocial impact of internal bleaching procedures as well as the effectiveness of intracoronary bleaching with two different bleaching agents (35\% hydrogen peroxide and 37\% carbamide peroxide) up to 3 months after treatment.

We found that both gels were highly effective in the technical walking bleach applications of non-vital teeth; the color achieved was stable 3 -month post-treatment. The patients reported a positive effect on aesthetic perception as well as psychosocial impact. Therefore, both hypotheses were correct because both gels were effective according to the pre-determined objective measurements. There were similar positive effects on aesthetic perception and psychosocial impact 3 months later.

Bleaching non-vital teeth is a minimally invasive treatment option that provides good clinical effectiveness and color/tone stability over time [15]. The results of the current study demonstrate the stability of treatment outcomes over 3 months following the use of the two most common gels with an objective methodology and trial design.

Regarding the OHIP, a statistically significant decrease was observed in total score 3-month post-whitening compared with the baseline measurements. This decrease indicates that non-vital tooth bleaching produces a substantial improvement in self-perception of patients and a noticeable reduction in the dimensions of functional limitation, physical pain and psychological discomfort. These values decreased significantly with the treatment and provide critical biopsychosocial implications because disadvantages experienced by a person due to cosmetic dental problems may profoundly affect their self-esteem, interactions, environmental adaptations, personal relationships, job opportunities, and other fundamental aspects affecting their quality of life [16].

Regarding functional limitation, positive effects were measured 3-month post-whitening. This finding reveals the effect of tooth whitening on functional limitation and suggests that the positive change on dental aesthetics enables patients to improve their interactions with their environment and enhance their interpersonal relationships due to this positive change [17]. The improvement in the psychological discomfort dimension was observed 1 week post-treatment and persisted throughout all of the subsequent evaluations. These improvements are consistent with the results of study from 2015 that noted improvements in the different dimensions of the aesthetic OHIP in response to tooth whitening [5]. Undoubtedly, patients perceived a positive psychological effect that was reflected in the score of this factor. They felt better with the color equalization of their teeth. Moreover, the positive psychological effect might have improved patients' perception of pain, which might have constituted a simple placebo effect because equalization of color has nothing to do with the etiopathogenesis of tooth sensitivity.

Our most important finding was the significant decrease in general score of OHIP, which was maintained for 3 months after treatment. We also noted an improvement in the aesthetic self-perception that persisted 3-month postbleaching. It would be interesting to follow this cohort of patients over time.

The PIDAQ measures three additional negative dimensions of psychosocial impact: social impact, psychological impact and aesthetic concern. Social impact aims to assess potential problems that an individual may face in social situations due to him or her having a subjectively unfavorable dental appearance. Psychological impact evaluates an individual's feelings of inferiority or unhappiness compared with others. Aesthetics concern includes data pertaining to the concern or disapproval that an individual's dental appearance generates when that individual looks in a mirror or views photographs or videos of him or herself [18]. Our results revealed a decrease in the scores of these three negative dimensions 3-month post-whitening compared with the baseline values. Therefore, intracoronal tooth whitening was able to generate a positive psychosocial effect over both the short term and the medium term. Hence, it is important to keep track of these treatments.

Comparing oneself to others can play an important role in psychosocial well-being, and feeling inferior to others can result in dysphoric states [13]. Our work has shown that there is an improvement in psychological well-being after tooth whitening and that this effect persists over time. Whitening improves patients' self-satisfaction; patients feel better and safer when they are pleased with the color of their teeth. The tools used in this study (PIDAQ and OHIP-14) confirmed that there are positive changes in both the psychosocial well-being of patients and the self-perception of cosmetic dentistry after tooth whitening and that these effects persist over the short term.

Many studies have shown that patients consider tooth color to be a factor in determining their satisfaction with their dental appearance $[1,19]$. Therefore, tooth discoloration can decrease a patient's self-fulfillment, resulting in detrimental effects to the patient's emotional state [20]. Moreover, according to the results of this study, it can be assumed that the discoloration of just one tooth can critically 
influence a patient's satisfaction with his or her appearance. Conversely, whitening that tooth not only influences the patient's satisfaction with his or her appearance but also positively affects their self-perception and promotes psychological well-being.

The available literature on the self-perception of aesthetics and psychosocial impact generated by tooth whitening is limited. There is more literature in the area of orthodontics $[18,20]$. Therefore, more research is needed to support the findings related to self-perception and the psychosocial impact of tooth whitening. Undoubtedly, the effect of having a dark tooth in the front of one's mouth generates a larger negative impact on the life of patients than does having a dark tooth in the back of one's mouth (i.e., on the premolars or premolars). In this study, we only bleached the front teeth (incisors) because the appearance of these teeth in an important factor determining the quality of life of people. Smile and color are factors that impact the well-being of individuals [3].

There have been little data reported regarding the "minimal important changes" (MIC) in the values of the two questionnaires. Hayran et al. [21] reported that a decrease of 3.5 points in the OHIP-14 was positively correlated with a clinical improvement in patients with Behçet's disease. In our study, there was a decrease of at least five points with respect to the baseline, which represents a larger change than that reported by Hayran et al. [21]. Thus, MIC provides interpretation guidelines for changes in scores from the patient perspective.

Concerning the variations in the PIDAQ values, there have been unfortunately no studies that have correlated these data with any actual clinical situation, unlike the case with the OHIP-14. However, considering the average values and the number of questions per assessed factor, it can be concluded that there was an improvement of at least one point for each factor. Considering that this questionnaire uses a five-point Likert scale (0-4), in terms of percentages it can be stated that the intracoronal bleaching at least had, on average, a quantitative influence of roughly $20 \%$ on the improvement in psychosocial impact.

There are several limitations associated with this study: difficult-to-control variables such as those related to clinical activity, patient drop-out, and many human-related factors. We also note the lack of negative control (patients without bleaching), which would have been useful for evaluating the questionnaires that measure psychosocial and aesthetic perception factors. Even so, we found significant differences in the analyses of quality of life, and we recommended carrying out studies with controls of parallel groups of patients that are not subjected to tooth whitening to evaluate the controlled effect on the quality of life.

In conclusion, intracoronal bleaching with either 35\% hydrogen peroxide or $37 \%$ carbamide peroxide generates a positive impact on aesthetic self-perception and psychosocial impact. The color change also persists for at least 3 months.

Funding This work was supported by the CONICYT - Fondecyt (Grant No. 1170575).

\section{References}

1. Samorodnitzky-Naveh, G. R., Geiger, S. B., \& Levin, L. (2007). Patients' satisfaction with dental esthetics. The Journal of the American Dental Association, 138(6), 805-808.

2. Plotino, G., Buono, L., Grande, N. M., Pameijer, C. H., \& Somma, F. (2008). Nonvital tooth bleaching: A review of the literature and clinical procedures. Journal of Endodontics, 34(4), 394-407.

3. Gupta, S. K., \& Saxena, P. (2014). Evaluation of patient satisfaction after non-vital bleaching in traumatized discolored intact anterior teeth. Dental Traumatology, 30(5), 396-399.

4. Fernandez, E., Bersezio, C., Bottner, J., Avalos, F., Godoy, I., Inda, D., Vildosola, P., Saad, J., Oliveira, O. B. Jr., \& Martin, J. (2017). Longevity, esthetic perception, and psychosocial impact of teeth bleaching by low (6\%) hydrogen peroxide concentration for in-office treatment: A randomized clinical trial. Operative Dentistry, 42(1), 41-52.

5. Martin, J., Vildosola, P., Bersezio, C., Herrera, A., Bortolatto, J., Saad, J. R., Oliveira, O. B. Jr., \& Fernandez, E. (2015). Effectiveness of $6 \%$ hydrogen peroxide concentration for tooth bleachingA double-blind, randomized clinical trial. Journal of Dentistry, 43(8), 965-972.

6. Meireles, S. S., Goettems, M. L., Dantas, R. V., Bona, A. D., Santos, I. S., \& Demarco, F. F. (2014). Changes in oral health related quality of life after dental bleaching in a double-blind randomized clinical trial. Journal of Dentistry, 42(2), 114-121.

7. Attin, T., Paque, F., Ajam, F., \& Lennon, A. M. (2003). Review of the current status of tooth whitening with the walking bleach technique. International Endodontic Journal, 36(5), 313-329.

8. Sarkis-Onofre, R., Cenci, M. S., Demarco, F. F., Lynch, C. D., Fleming, P. S., Pereira-Cenci, T., \& Moher, D. (2015). Use of guidelines to improve the quality and transparency of reporting oral health research. Journal of Dentistry, 43(4), 397-404.

9. World Medical Association Declaration of Helsinki. (2002). Ethical principles for medical research involving human subjects. Nursing Ethics, 9(1), 105-109.

10. Faul, F., Erdfelder, E., Lang, A. G., \& Buchner, A. (2007). $\mathrm{G}^{*}$ Power 3: A flexible statistical power analysis program for the social, behavioral, and biomedical sciences. Behavior Research Methods, 39(2), 175-191.

11. Martin, J., Vildosola, P., Bersezio, C., Herrera, A., Bortolatto, J., Saad, J. R. C., Oliveira, O. B., \& Fernandez, E. (2015). Effectiveness of $6 \%$ hydrogen peroxide concentration for tooth bleachingA double-blind, randomized clinical trial. Journal of Dentistry, 43(8), 965-972.

12. Núñez, L., Dreyer, E., Martin, J., \& Moncada, G. (2013). Validation of the OHIP-aesthetic Sp Questionnaire for chilean adults. The Journal of Dental, Oral and Craniofacial Epidemiology, 1(2), 4-9.

13. Khan, M., \& Fida, M. (2008). Assessment of psychosocial impact of dental aesthetics. Journal of the College of Physicians and Surgeons Pakistan, 18(9), 559-564.

14. Montiel-Company, J. M., Bellot-Arcis, C., \& Almerich-Silla, J. M. (2013). Validation of the psychosocial impact of dental aesthetics questionnaire (Pidaq) in Spanish adolescents. Medicina Oral Patologia Oral y Cirugia Bucal, 18(1), e168-e173. 
15. Amato, M., Scaravilli, M. S., Farella, M., \& Riccitiello, F. (2006). Bleaching teeth treated endodontically: Long-term evaluation of a case series. Journal of Endodontics, 32(4), 376-378.

16. Wong, A. H., Cheung, C. S., \& McGrath, C. (2007). Developing a short form of ORAL Health Impact Profile (OHIP) for dental aesthetics: OHIP-aesthetic. Community Dentistry and Oral Epidemiology, 35(1), 64-72.

17. Davis, L. G., Ashworth, P. D., \& Spriggs, L. S. (1998). Psychological effects of aesthetic dental treatment. Journal of Dentistry, 26(7), 547-554.

18. Klages, U., Bruckner, A., \& Zentner, A. (2004). Dental aesthetics, self-awareness, and oral health-related quality of life in young adults. European Journal of Orthodontics, 26(5), 507-514.
19. Onyeaso, C. O., Utomi, I. L., \& Ibekwe, T. S. (2005). Emotional effects of malocclusion in Nigerian orthodontic patients. The Journal of Contemporary Dental Practice, 6(1), 64-73.

20. de Oliveira, C. M., \& Sheiham, A. (2004). Orthodontic treatment and its impact on oral health-related quality of life in Brazilian adolescents. Journal of Orthodontics, 31(1), 20-27. (discussion 15).

21. Hayran, O., Mumcu, G., Inanc, N., Ergun, T., \& Direskeneli, H. (2009). Assessment of minimal clinically important improvement by using Oral Health Impact Profile-14 in Behcet's disease. Clinical and Experimental Rheumatology, 27(2 Suppl 53), S79-S84. 